\title{
Assessment of Visual and Chromatic Functions in a Rodent Model of Retinal Degeneration
}

\author{
Francisco Segura,,${ }^{1,2}$ Ana Sánchez-Cano, ${ }^{2,3}$ Sebastián Jarabo, ${ }^{3}$ Carmen López de la Fuente, ${ }^{2,3}$ \\ Nicolás Cuenca, ${ }^{4}$ María P. Villegas-Pérez, ${ }^{5}$ and Isabel Pinilla ${ }^{1,2,6}$ \\ ${ }^{1}$ Department of Surgery, Gynecology and Obstetrics, University of Zaragoza, Zaragoza, Spain \\ ${ }^{2}$ Aragon Institute for Health Research (IIS Aragón), Aragón, Spain \\ ${ }^{3}$ Department of Applied Physics, University of Zaragoza, Zaragoza, Spain \\ ${ }^{4}$ Laboratory of the Neurobiology of the Visual System, Department of Physiology Genetics and Microbiology, University of Alicante, \\ Alicante, Spain \\ ${ }^{5}$ Department of Ophthalmology, University of Murcia, Murcia, Spain \\ ${ }^{6}$ Department of Ophthalmology, Lozano Blesa University Hospital, Zaragoza, Spain
}

Correspondence: Isabel Pinilla, Department of Ophthalmology, Lozano Blesa University Hospital, San Juan Bosco 15, 50009 Zaragoza, Spain; isabel.pinilla@telefonica.net.

Submitted: May 12, 2015

Accepted: August 18, 2015

Citation: Segura F, Sánchez-Cano A, Jarabo S, et al. Assessment of visual and chromatic functions in a rodent model of retinal degeneration. Invest Ophthalmol Vis Sci. 2015;56:62756283. DOI:10.1167/iovs.15-17257
Purpose. We evaluated the photoreceptor response of pigmented $\mathrm{P} 23 \mathrm{H}$ and normal pigmented Long Evans (LE) rats over time using functional tests in variable lighting conditions.

Methods. Pigmented $\mathrm{P} 23 \mathrm{H}$ rats were studied by optomotor testing and electroretinogram (ERG) recordings at P30, P150, and P240. Pigmented LE rats were used as a normal wild-type control. Stimuli were modified with colored filters. Neutral density filters were used to reduce luminance.

Results. Age-related decreases in visual acuity (VA) and contrast sensitivity (CS) were observed in $\mathrm{P} 23 \mathrm{H}$ rats. Good correlations in measurements without filter and with green filter were observed between LE and P23H P30 rat values. Differences between groups were smaller with red and purple filters. A strong relationship with luminance was observed in LE rats (VA and CS) and with $\mathrm{P} 23 \mathrm{H} \mathrm{P} 30$ rats (CS). A decline in the ERG responses of $\mathrm{P} 23 \mathrm{H}$ rats was consistent with the gradual loss of photoreceptors. Differences in a- and b-wave amplitudes with different colored filters were negligible with the exception of the red filter, which resulted in smaller responses.

Conclusions. Visual function parameters decreased with age in pigmented $\mathrm{P} 23 \mathrm{H}$ rats. Irrespective of luminance, color filter, and retinal degeneration, minimum thresholds of VA and CS were found. Smaller differences than expected were found using color filters. Responses to functional tests at long wavelengths were observed, where there is very low photoreceptor spectral sensitivity. The use of filters with functional testing could minimize light-induced retinal damage in rats.

Keywords: $\mathrm{P} 23 \mathrm{H}$, retinitis pigmentosa, optokinetic tracking, electroretinogram
$R$ ats are nocturnal animals with a rod-dominated retina. However, their retina also contains a small number of cones. ${ }^{1}$ Rats maintain an order of magnitude difference in the numbers of cones with maximum sensitivity in the short wavelength (S-cones, with a peak of $358 \mathrm{~nm}$ ) compared to the middle and long wavelengths (ML-cones, peaking at approximately $509 \mathrm{~nm})^{2,3}$ Middle and long wavelength cones constitute approximately $90 \%$ of cones; the other $10 \%$ are Scones that are thought to be UV-sensitive elements, but they probably do not appreciably contribute to the photopic system of the rat. ${ }^{4}$ The maximum responses of the two types of cones and rods of rats have been widely studied by electroretinography (ERG), however the contribution of each type of cone to the visual system is not clear. ${ }^{3,5}$

Retinitis pigmentosa (RP) is the most common form of inherited photoreceptor degeneration. ${ }^{6,7}$ It comprises a group of diseases characterized by a progressive anatomical and functional loss of rod and cone photoreceptors. ${ }^{8}$ Autosomal dominant RP is responsible for most $\mathrm{RP}$ cases, ${ }^{9}$ and most cases are due to rhodopsin mutations. Cones are lost after the rod degeneration. The transgenic albino $\mathrm{P} 23 \mathrm{H}$ rat is a well-studied model of autosomal dominant RP. In pigmented RP rodent models, a progressive deterioration also occurs in retinal function and anatomy. Because an important role of retinal pigmentation is to prevent light damage, we studied heterozygous pigmented $\mathrm{P} 23 \mathrm{H}$ rats. These animals undergo slower retinal degeneration, provide a closer model to human RP, and allow straightforward evaluation by functional testing. ${ }^{10}$

Retinal damage related to exposure to intense visible light has been studied widely. ${ }^{11,12}$ The retinas of genetically inbred albino rodents have been shown to be particularly susceptible to photic injury induced by moderate and high levels of light exposure. ${ }^{13-15}$ Not all rhodopsin mutations seem to have the same susceptibility to light damage. Other genetic factors also have been reported to be involved in the light sensitivity of different rodent strains. ${ }^{13,14,16}$ There is a direct relationship between light and retinal damage, so that a longer duration of exposure or higher intensity of light results in greater retinal 


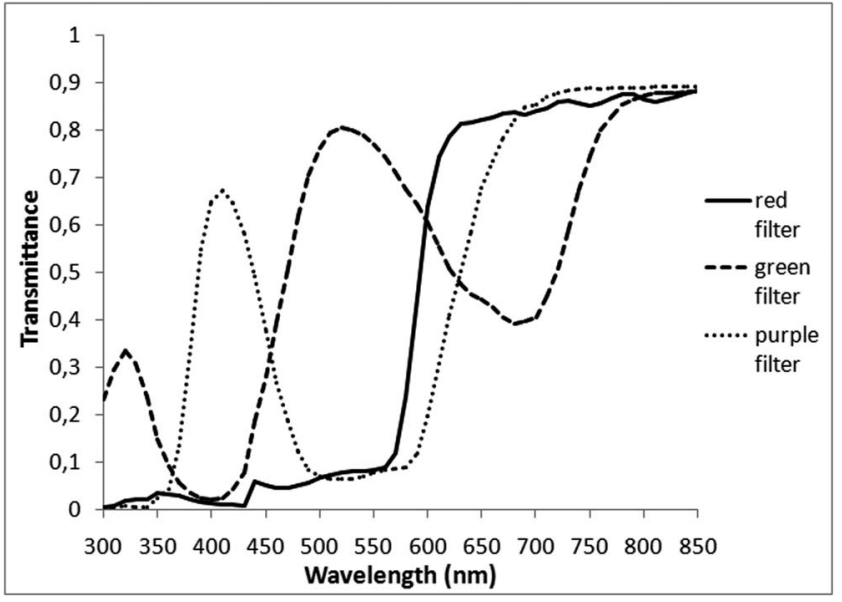

Figure 1. Curves of transmittance of colored filters from 300 to 850 nm. Red, continuous line; green, dashed line; purple, dotted line.

damage. ${ }^{17}$ Using measurement techniques that involve less exposure to light could assess the contribution of the different photoreceptors and minimize damage related to light on the retina of animal models.

Electroretinographic evaluation is one of the most widely used methods to determine visual function in animal models. ${ }^{10,18-20}$ The introduction of functional devices, such as the OptoMotry system (OptoMotry; CerebralMechanics, Lethbride, Alberta, Canada), ${ }^{21,22}$ a noninvasive method used to examine the response of the rat in vivo, allows for the study of cone and rod degeneration over time and allows for the in vivo evaluation of therapeutic interventions in the same animal. Optokinetic tracking (OKT) measurements have the advantage of robust behavior and do not require the rat to be trained, allowing a rapid evaluation of visual features, such as visual acuity (VA) and contrast sensitivity (CS). ${ }^{10,23-26}$

The aim of our study was to evaluate the photoreceptor response of pigmented $\mathrm{P} 23 \mathrm{H}$ and pigmented normal Long Evans (LE) rats over time, and to identify the relative contributions of rods and cones using functional tests in different lighting conditions.

\section{MeTHODS}

\section{Animals}

Pigmented transgenic rats, heterozygous for the $\mathrm{P} 23 \mathrm{H}$ rhodopsin mutation, were bred from a cross between normal pigmented LE rats and transgenic albino homozygous $\mathrm{P} 23 \mathrm{H}$ line 1 . Animals were studied by optomotor testing and ERG recordings at P30, P150, and P240. Long Evans rats (P90) were used as the wildtype control. Eight animals of each group were studied.

Transgenic rats were obtained from Dr M. LaVail (University of California, San Francisco, San Francisco, CA, USA), bred in a colony at the University of Zaragoza, Zaragoza, Spain, and maintained under a 12-hour light/dark cycle. Animals were housed and handled with the authorization and supervision of the Institutional Animal Care and Use Committee from the University of Zaragoza. Procedures were performed in accordance with the ARVO Statement for the Use of Animals in Ophthalmic and Vision Research.

\section{Visual Acuity and Contrast Sensitivity Evaluation}

To evaluate visual parameters, 8 pigmented $\mathrm{P} 23 \mathrm{H}$ rats were measured at P30, P150, and P240. Eight LE rats were evaluated at P90. The assessment of VA and CS was performed using an OptoMotry system (OptoMotry, CerebralMechanics). ${ }^{21,22}$ The device consists of a testing chamber created with four screens facing into a square. Animals were placed on a platform in the center of the square. A virtual cylinder comprised of a vertical sine wave grating was projected in $3 \mathrm{D}$ coordinate space and rotated around the animal. A video camera, situated above the animal, provided real time feedback on another screen. Rats were allowed to move freely on the platform, and the spatial frequency of the grating was maintained at the animal's viewing position by recentering the cylinder on the rat's head. The cylinder was rotated at a constant speed $\left(12^{\circ} / \mathrm{s}\right)$.

The experimenter judged whether the rats made tracking motions with reflexive head and neck movements following the stimulus. Animals were assessed for tracking behavior for 5 seconds, and then a gray stimulus appeared, to reduce the possibility of adapting to the stimulus. Spatial frequency thresholds were calculated by systematically increasing the spatial frequency of the grating at $100 \%$ contrast until the animals no longer responded. This threshold was considered the maximum VA. A CS curve was generated by identifying the minimum contrast that generates tracking over a range of spatial frequencies.

\section{Filter Specifications}

The grating was modified with colored filters (green, red, and purple) placed in front of the screens. Transmittance of the filters from 300 to $850 \mathrm{~nm}$ was measured in a spectrophotometer, and curves of transmittance were plotted (Fig. 1). The total visible transmittance was $69.82 \%, 23.56 \%$, and $13.76 \%$ for the green, red, and purple filter, respectively. Neutral density (ND) $12 \%$ filters were used in combination with colored filters to reduce the luminance. In that case, transmittance was $9.61 \%, 2.54 \%$, and $1.46 \%$, respectively.

Colored filters were selected as a consequence of the peaks of maximum response in S-cones (358 nm) and ML-cones (509 $\mathrm{nm})$. The green filter was selected with the closest curve of transmittance to the ML-cones. ${ }^{27}$ The purple filter allowed a good transmission between 370 and $480 \mathrm{~nm}$, and after $590 \mathrm{~nm}$, to minimize the ML-cone response. The red filter gave a good value of transmittance only after $570 \mathrm{~nm}$.

\section{ERG Recordings}

Pigmented $\mathrm{P} 23 \mathrm{H}$ rats were studied by ERG recordings at $\mathrm{P} 30$, $\mathrm{P} 150$, and P240. Eight animals were studied for each time point. Eight normal LE rats at age P90 were used as wild-type controls. In both groups, four animals were tested with each colored filter and another four rats without filters.

Rats were adapted to darkness overnight and prepared for recording under a dim red light. Animals were anesthetized with an intraperitoneal injection of a mixture of ketamine (90 $\mathrm{mg} / \mathrm{kg})$ and xylazine $(10 \mathrm{mg} / \mathrm{kg})$, and maintained on a heating pad at a stable temperature. The ketamine/xylazine anesthetic shows robust ERGs with large a-wave and b-wave amplitudes, and low eye movements. ${ }^{28}$ Pupils were dilated by applying a topical drop of $1 \%$ tropicamide (Colircusí Tropicamida; Alcon, Barcelona, Spain). A topical drop of 2\% Methocel (OmniVision, Puchheim, Germany) was instilled in each eye before situating the corneal electrode. Furthermore, a drop of $0.9 \%$ saline was applied occasionally to the cornea to prevent dehydration and to allow electrical contact with the recording electrode (gold wire loop). Two 25-gauge platinum needles inserted under the scalp, behind the eyes, served as the reference electrodes, with a ground electrode located in the tail. All experiments were performed in absolute darkness. Stimulus presentation and 


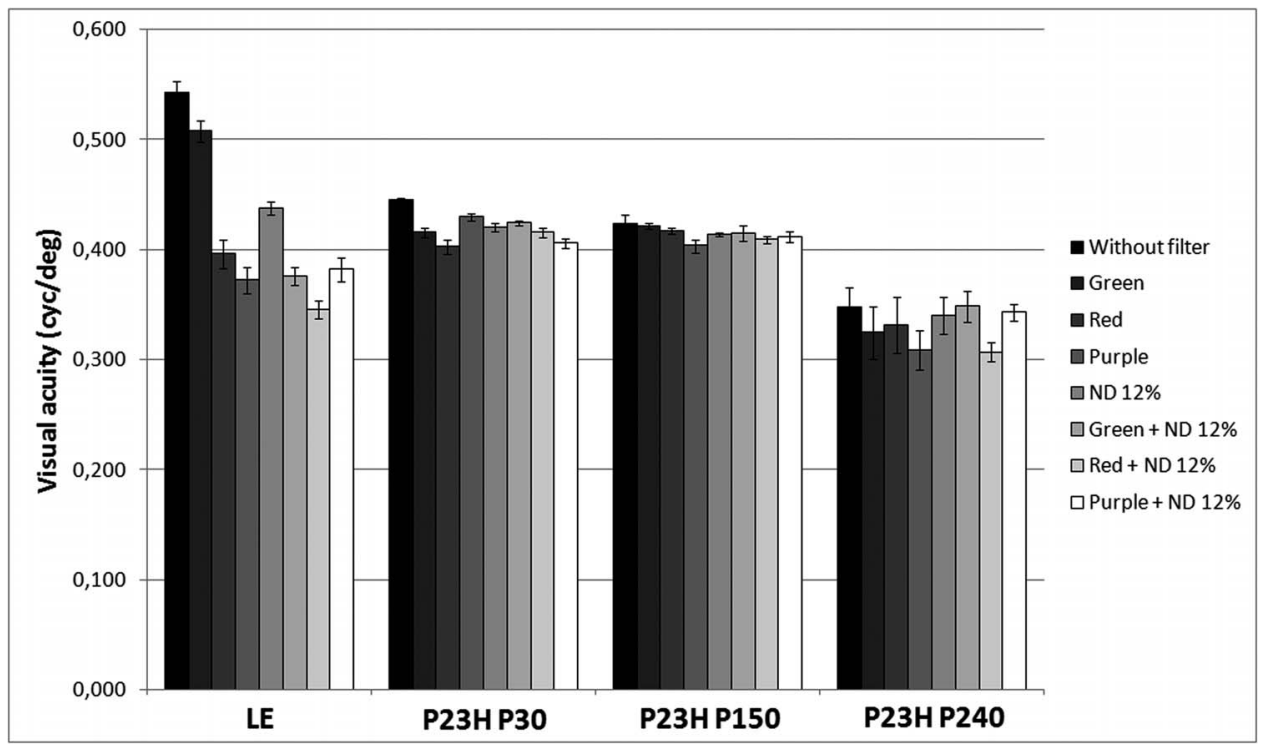

FIGURE 2. Visual acuity of LE (P90) and P23H rats (P30, P150, and P240) using different filters. Error bars: SEM.

data acquisition were provided by the Espion system from Diagnosys LLC (Cambridge, UK).

\section{Mixed b-Wave}

Dark-adapted b-waves show aggregate rod and cone pathway contributions. To describe them, recordings of 3 to 8 single flash presentations of $10 \mu$ s duration were displayed. Stimuli were presented at 10 increasing intensities varying from -3.70 to 2.86 $\log \mathrm{cd} / \mathrm{m}^{2}$ in luminance. Interstimuli intervals (ISI) were increased to minimize the effects of bleaching on the rods, which could reduce the b-wave amplitude during successive flashes. The ISI was elevated from 10 seconds at lowest stimulus intensity $(-3.70$ $\left.\log \mathrm{cd} / \mathrm{m}^{2}\right)$ up to 120 seconds at highest stimulus intensity (2.86 $\log \mathrm{cd} / \mathrm{m}^{2}$ ). The amplitude of the a-wave was measured from the baseline to the trough of the a-wave. The amplitude of the b-wave was measured from the trough of the a-wave up to the peak of the b-wave. The results of $a$ - and b-waves were averaged for different recordings. To determine if the ERG response remained, criterion amplitudes were established at $20 \mu \mathrm{V}$ for a- and b-waves.

\section{Isolation of the Cone Response Using a Double Flash Protocol}

The double flash protocol was similar to previous studies. ${ }^{29} \mathrm{~A}$ probe flash was presented 1 second after a conditioning flash. The role of the first flash is to temporarily saturate rods so that they do not respond to the probe flash. The necessary intensity of the conditioning flash for complete rod bleaching was set to 1.4 $\log \mathrm{cd} / \mathrm{m}^{2}$. The probe flash intensity also was $1.4 \log \mathrm{cd} / \mathrm{m}^{2}$. The response to the probe flash, preceded by the conditioning flash, was taken as a reflection of cone-driven activity. A rod-driven bwave was obtained by subtracting the cone-driven response from the mixed response (obtained by the conditioning flash alone). The results were averaged for 3 recordings, with an ISI of 100 seconds to assure full recovery of rod responsiveness

\section{Results}

\section{VA and CS Evaluation}

In $\mathrm{P} 23 \mathrm{H}$ rats, VA was progressively lost with age as shown in the measurements without filters (Fig. 2). Visual acuity values were $0.542 \pm 0.011$ cycles/deg for LE and $0.445 \pm 0.002$ cycles/deg for $\mathrm{P} 23 \mathrm{H}$ at $\mathrm{P} 30$, and $0.424 \pm 0.008$ cycles/deg at $\mathrm{P} 150$ and $0.347 \pm 0.019$ cycles/deg at P240. In the LE group, smaller values with the green filter $(0.508 \pm 0.010$ cycles $/ \mathrm{deg})$ and even smaller values with the other filters $(0.438 \pm 0.006$ cycles/deg for ND $12 \%$ to $0.346 \pm 0.008$ cycles/deg for red + ND 12\%) were observed. Smaller differences were found in the $\mathrm{P} 23 \mathrm{H}$ groups between measured values without a filter and the worst value from colored filters $(0.042$ cycles/deg at P30, 0.020 cycles/deg at P150, and 0.040 cycles/deg at P240).

A similar trend was found in CS curves (Fig. 3). In the LE rats, peaks of $52.63 \pm 5.43$ (with no filter) and $35.13 \pm 1.23$ (ND 12\% filter) were obtained for a spatial frequency of 0.089 cycles/deg. The filter addition gave smaller values, between $26.99 \pm 1.60$ and $18.36 \pm 0.64$. The behavior was different with the green filter, which gave similar values to those obtained without filters, $49.29 \pm 2.87$. Similar results were found in the $\mathrm{P} 23 \mathrm{H}$ groups. Peaks of $39.86 \pm 0.85$ and $33.87 \pm$ 0.94 (P30), $31.11 \pm 1.16$ and $25.99 \pm 1.21$ (P150), and 31.54 \pm 1.78 and $26.81 \pm 1.97$ (P240) were obtained for measurements without filters and with the ND $12 \%$ filter, respectively. Colored filters gave smaller values, between 24.47 \pm 0.95 and $17.99 \pm 0.57(\mathrm{P} 30$, excluding the values obtained with the green filter that were similar to those obtained without filters), $24.95 \pm 1.09$ and $18.38 \pm 0.89$ (P150), and $21.72 \pm 1.28$ and $12.17 \pm 0.77$ (P240). As expected, LE rats had better results in all cases. Similar results without filters and with green filters were observed between LE and $\mathrm{P} 23 \mathrm{H} \mathrm{P} 30$ rat values. Differences between groups were lower with red and purple filters.

\section{Relationship Between Visual Parameters and Luminance}

Luminance of the screens with different filters was measured by a luminance meter LP 471 Lum 2 (Delta Ohm, Padua, Italy). Visual acuity (Fig. 4A) and CS results (Fig. 4B) were represented as a function of luminance. The LE rats' VA showed a strong relationship with luminance, achieving an $R^{2}$ value of 0.94 . No relationships were found in the $\mathrm{P} 23 \mathrm{H}$ rat groups, with $R^{2}$ values $<0.7$. Furthermore, a relationship of CS with luminance also was observed in the LE rats $\left(R^{2}=0.91\right)$ 

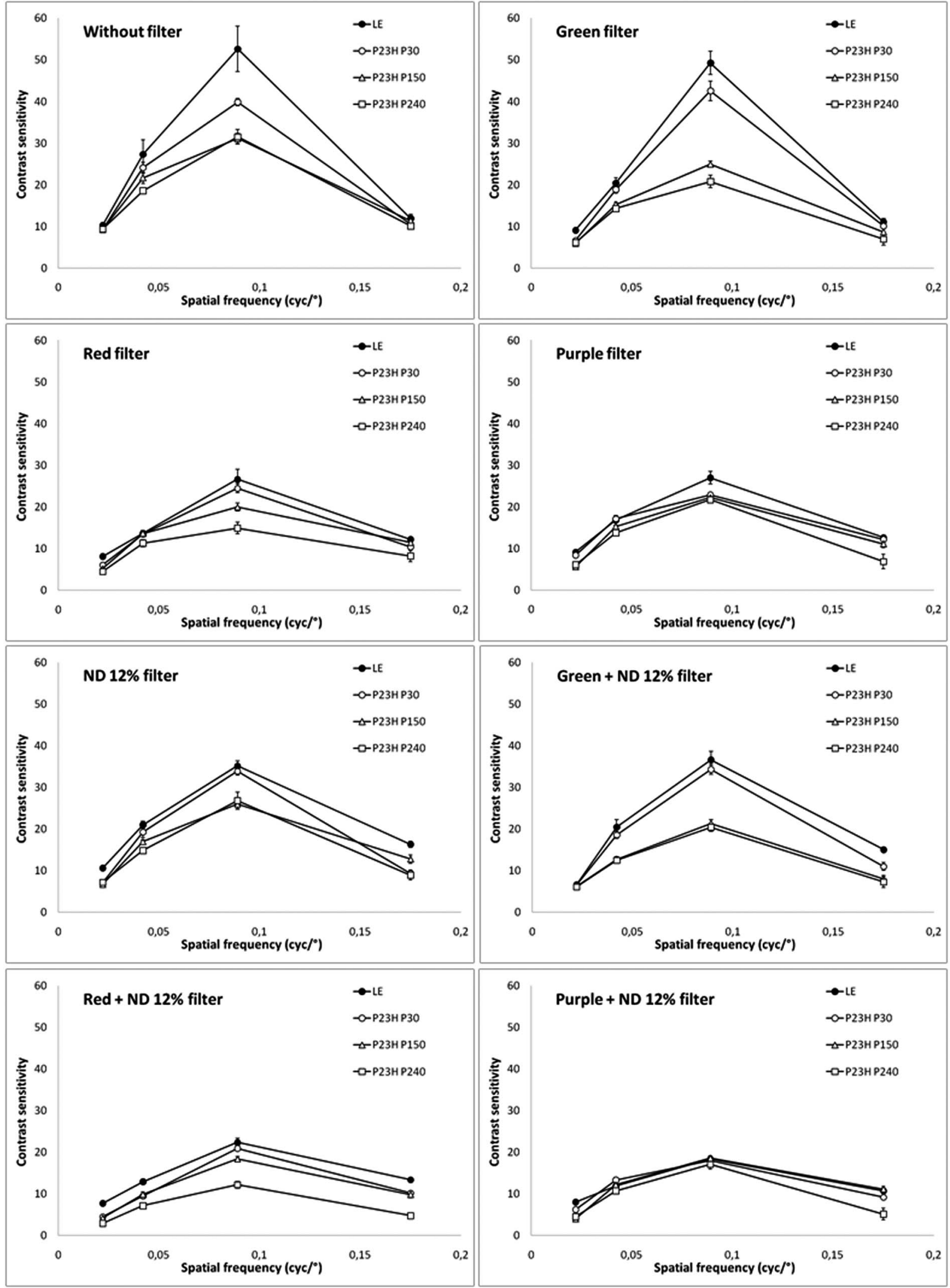

Figure 3. Contrast sensitivity as a function of spatial frequency with colored filters and adding an ND filter (ND 12\%). Measures of the wild-type control LE (black circle), P23H at P30 (white circle), P150 (triangle), and P240 (square) were done. Each point represents the mean of 8 animals. Error bars: SEM. 

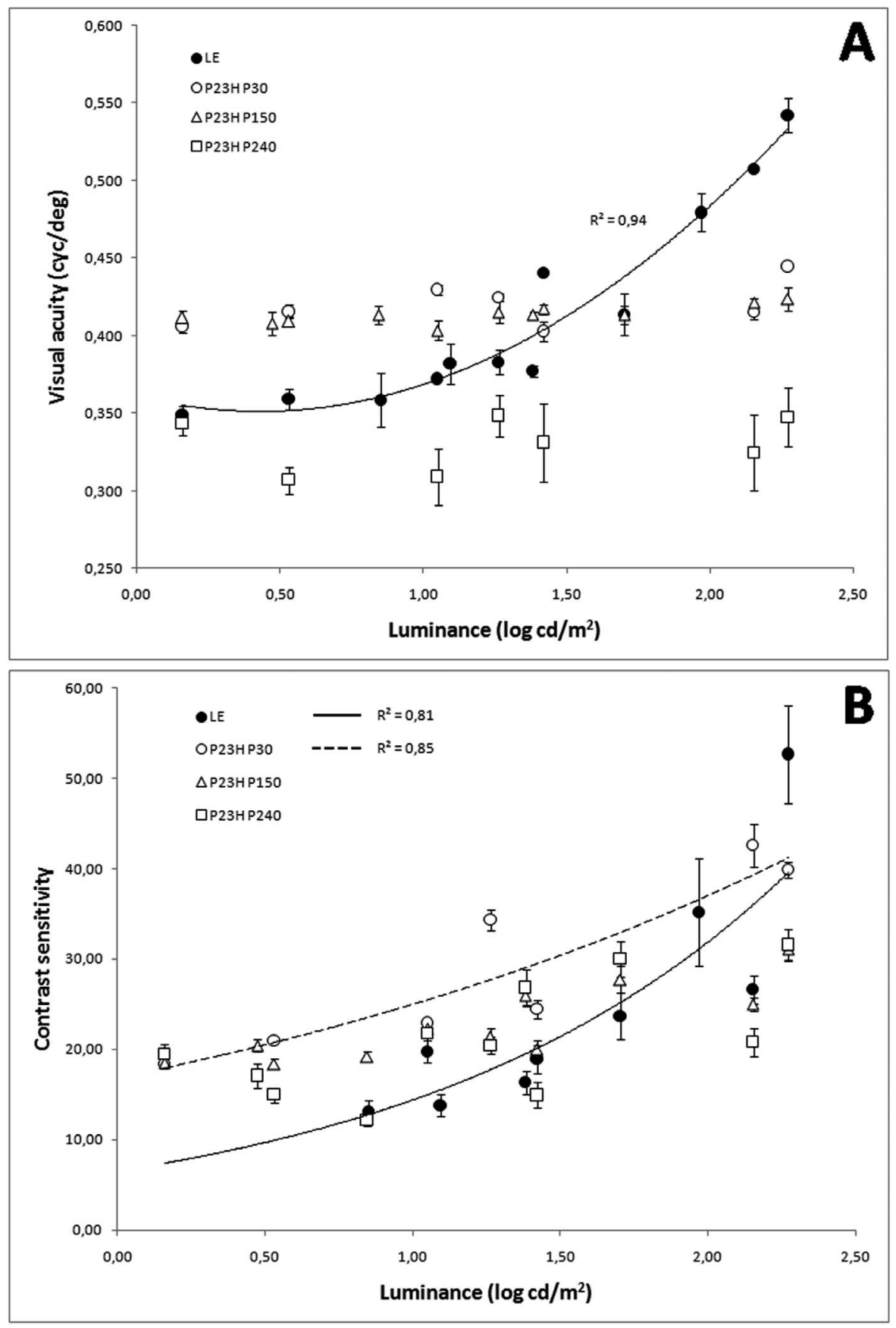

Figure 4. Visual acuity (A) and contrast sensitivity (B) as a function of luminance from normal LE rats (black circle) recorded at age P90 and from pigmented $\mathrm{P} 23 \mathrm{H}$ rats recorded at P30 (white circle), P150 (triangle), and P240 (square). Each point represents the mean of 8 rats. Vertical bars: SEM. Lines of fit with $R^{2}>0.8$ are plotted: $R^{2}=0.94$ in the LE group for VA (A), $R^{2}=0.91$ and $R^{2}=0.85$ in LE rats (continuous line) and the P23H group at P30 (dashed line), respectively, for contrast sensitivity (B).

and the $\mathrm{P} 23 \mathrm{H}$ rats at $\mathrm{P} 30\left(R^{2}=0.85\right)$, and a lower relationship was found at P150 $\left(R^{2}=0.73\right)$.

\section{ERG Recordings}

In pigmented $\mathrm{P} 23 \mathrm{H}$ rats, the scotopic a-wave (Fig. 5A) reached a maximum value of $113 \pm 10 \mu \mathrm{V}$ at $\mathrm{P} 30$. This value represents $37 \%$ of the same value in normal $\mathrm{LE}$ rats $(304 \pm 34 \mu \mathrm{V})$. Despite being affected at early ages, a-waves from $\mathrm{P} 23 \mathrm{H}$ rats still could be evoked to P150 $(25 \pm 5 \mu \mathrm{V})$, but they were almost negligible at P240 $(<20 \mu \mathrm{V})$, the latest age studied. At all ages of $\mathrm{P} 23 \mathrm{H}$ rats, b-waves (Fig. 5B) were less affected than awaves, reaching maximum values of $1092 \pm 38 \mu \mathrm{V}$ (P30), 475 $\pm 45 \mu \mathrm{V}(\mathrm{P} 150)$, and $301 \pm 21 \mu \mathrm{V}$ (P240) with regard to 1178 $\pm 101 \mu \mathrm{V}$ (LE at P90).

Figure 5C illustrates the results of the ERG recordings obtained by the application of the double flash protocol from control LE (P90) and $\mathrm{P} 23 \mathrm{H}$ rats at $\mathrm{P} 30, \mathrm{P} 150$, and $\mathrm{P} 240$. The first flash elicited a mixed rod-cone response (left traces), and the second flash (one second delay) elicited a pure cone response (middle traces). The right traces were calculated by subtracting the middle traces from the left traces to obtain the 


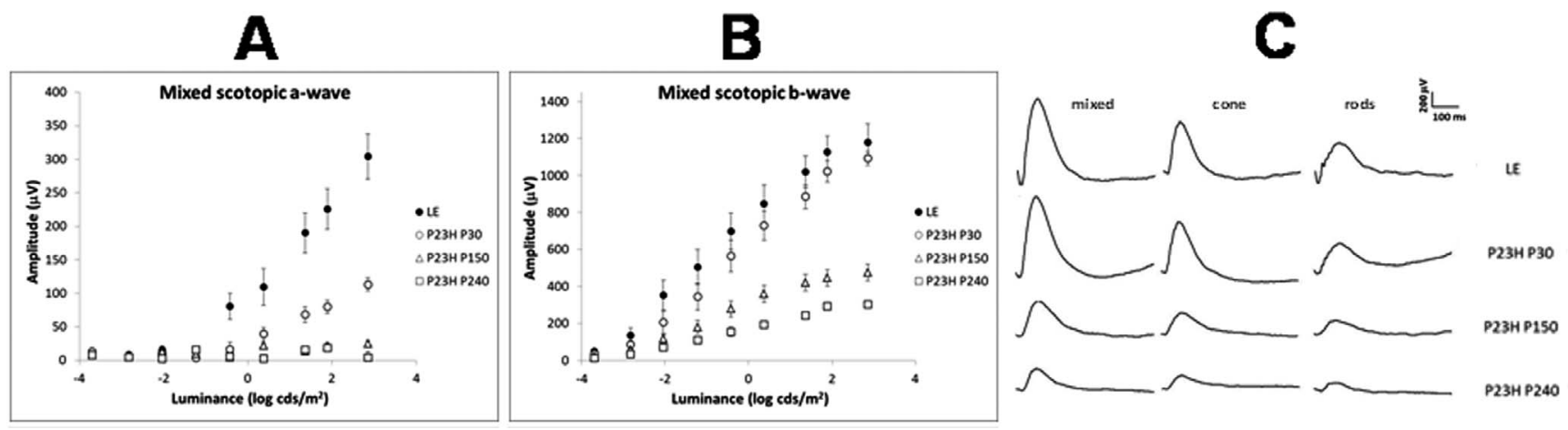

FiguRE 5. Electroretinography amplitude versus luminance series of mixed scotopic a-waves (A) and of mixed scotopic b-waves (B) from normal LE rats (black circle) recorded at $\mathrm{P} 90$, and from pigmented $\mathrm{P} 23 \mathrm{H}$ rats recorded at $\mathrm{P} 30$ (white circle), $\mathrm{P} 150$ (triangle), and $\mathrm{P} 240$ (square). Each point represents the mean of 8 animals. Vertical bars: SEM. (C) Application of the double flash protocol in LE (P90) and P23H rats (P30, P150, and P240). Left traces: mixed response obtained with a conditioning flash alone. Middle traces: isolated cone response obtained with a probe flash preceded by the conditioning flash. Right traces: rod response obtained by subtracting the isolated cone response from the mixed b-wave. Stimulus presentation is at the beginning of each trace. Each trace represents the mean of 8 rats.

rod-driven contribution to the ERG response. The rod-driven responses were affected by age in the $\mathrm{P} 23 \mathrm{H}$ rats: $\mathrm{a}$ - and $\mathrm{b}$-waves reached values of 40 and $391 \mu \mathrm{V}$ at $\mathrm{P} 30,<20$ and $188 \mu \mathrm{V}$ at $\mathrm{P} 150$, and 32 and $109 \mu \mathrm{V}$ at P240, compared to 165 and $516 \mu \mathrm{V}$ in the $\mathrm{LE}$ rats. The rod-driven contribution to the mixed scotopic b-waves was higher in LE rats (46\%) compared to $\mathrm{P} 23 \mathrm{H}$ rats, remaining stable for all ages $(38 \%$ at $\mathrm{P} 30,40 \%$ at $\mathrm{P} 150$, and $37 \%$ at $\mathrm{P} 240$ ).

Figure 6 shows the ERG amplitudes as a function of luminance and the results of the double flash protocol from the $\mathrm{LE}$ (P90) and $\mathrm{P} 23 \mathrm{H}$ rats (P30, P150, and $\mathrm{P} 240)$, measured with different colored filters. In the $\mathrm{P} 23 \mathrm{H}$ group, similar results in the $a-$ and b-wave values were obtained by adding different filters (Figs. 6A, 6B), excluding the P30 a-wave (value changed from $65 \mu \mathrm{V}$ with the red filter to $152 \mu \mathrm{V}$ without the filter) and the b-wave at P150 (from $309 \mu \mathrm{V}$ without the filter to $608 \mu \mathrm{V}$ with the green filter). Larger differences were observed in LE rats, but we were not able to identify any pattern between aand b-waves and colored filters (Figs. 6A, 6B).

There were differences in the a-waves of rod-driven responses in all groups (Fig. 6C). A-waves from $\mathrm{P} 23 \mathrm{H}$ rats were negligible $(<20 \mu \mathrm{V})$ when using red and purple filters for all ages. In LE rats, the results were smaller with the same filters (52 $\mu \mathrm{V}$ for red and $160 \mu \mathrm{V}$ for purple, compared to $242 \mu \mathrm{V}$ for green and $266 \mu \mathrm{V}$ without a filter). No clear relationship between the b-wave of rod-driven responses and colored filters was found. However, there were differences in the rod-driven contribution to mixed scotopic b-waves, with a higher percentage using green filters (more than $40 \%$ in all cases) and no filters (more than 50\%) compared to red and purple filters (less than 40\% and, except one case, less than 33\%).

\section{Discussion}

The present study has applied functional testing (OKT and ERG) to an animal model of progressive retinal degeneration (pigmented $\mathrm{P} 23 \mathrm{H}$ rat) and a wild-type control (LE rat) to evaluate the photoreceptor response, and the relative contributions of rods and cones in different lighting conditions. To our knowledge, there have been no examples of using these functional tests with in vivo photoreceptor responses and adding color filters to isolate the different photoreceptor responses.

It long has been known that rodents have two types of cones. ${ }^{30}$ Most cone photoreceptors are ML-cones, responsible for the green photopic sensitivity of the rat. The role of the S- cones under photopic lighting conditions has been less studied. Based on the peaks of maximum absorbance of cones and rods, we selected colored filters with a specific spectral sensitivity curve (Fig. 1) for isolating the responses of different photoreceptors in vivo. Using this method, new information could be obtained about the changes in the different cone photoreceptors following the rod loss in this autosomal model of rhodopsin gene mutation. Cone evaluation and preservation should be one of the main issues after therapeutic approaches.

Comparing the results obtained with age, a decrease in visual function (VA and CS) was observed in $\mathrm{P} 23 \mathrm{H}$ rats, consistent with the progressive cell loss in this retinal degeneration model. Thus, decreased values of VA (above 0.300 cycles/deg) and CS (above 30 , or $3.3 \%$ ) were found at advanced ages in degenerative rats. The late loss of cone function (compared to rods) in the rhodopsin mutation transgenic rat line ${ }^{31,32}$ could be an explanation of the results.

Considering the results given with colored filters (Fig. 2), differences of VA between measures without filters and the worst value with colored filters in the $\mathrm{P} 23 \mathrm{H}$ rats $(0.042$ cycles/ deg at P30, 0.020 cycles/deg at P150, and 0.040 cycles/deg at P240) were small. Larger differences were found in LE rats (from 0.542 cycles/deg without filters to 0.346 cycles/deg, the worst obtained value with colored filters). The same trend was observed in the CS curves (Fig. 3), although the differences were larger. With the choice of these filters, a good correlation between no filter and the green filter (as in LE and P23H P30 groups) would be expected, with no response or a very low response using the red and purple filters due to the possible contribution of S-cones in photopic vision. However, the minimum thresholds of VA (above 0.300 cycles/deg) and CS (above 12 , or $8.3 \%$ ) were obtained again. The minimum transmittance of the colored filters $(<7 \%$ at $509 \mathrm{~nm}$, peak of the M-cones) and that the light levels were within the photopic range (with a lowest luminance value of $1.5 \mathrm{~cd} / \mathrm{m}^{2}$ ) were enough to generate an OKT response, which could explain these results.

If VA and CS are plotted as a function of luminance (Fig. 4) as others have done, ${ }^{33}$ a good correlation is observed in LE rats for VA $\left(R^{2}=0.94\right)$ as CS $\left(R^{2}=0.91\right)$ and for CS $\left(R^{2}=0.085\right)$ in $\mathrm{P} 23 \mathrm{H}$ at $\mathrm{P} 30$ regardless of the colored filter used. A stronger relationship between OKT response and luminance in the slightly degenerate and nondegenerate retina could be an explanation for these results

Recently, it has been observed that modest increases in ambient light cause a rapid and significantly reversible loss of cone and rod function. ${ }^{34}$ Cones are damaged rapidly in the rat 


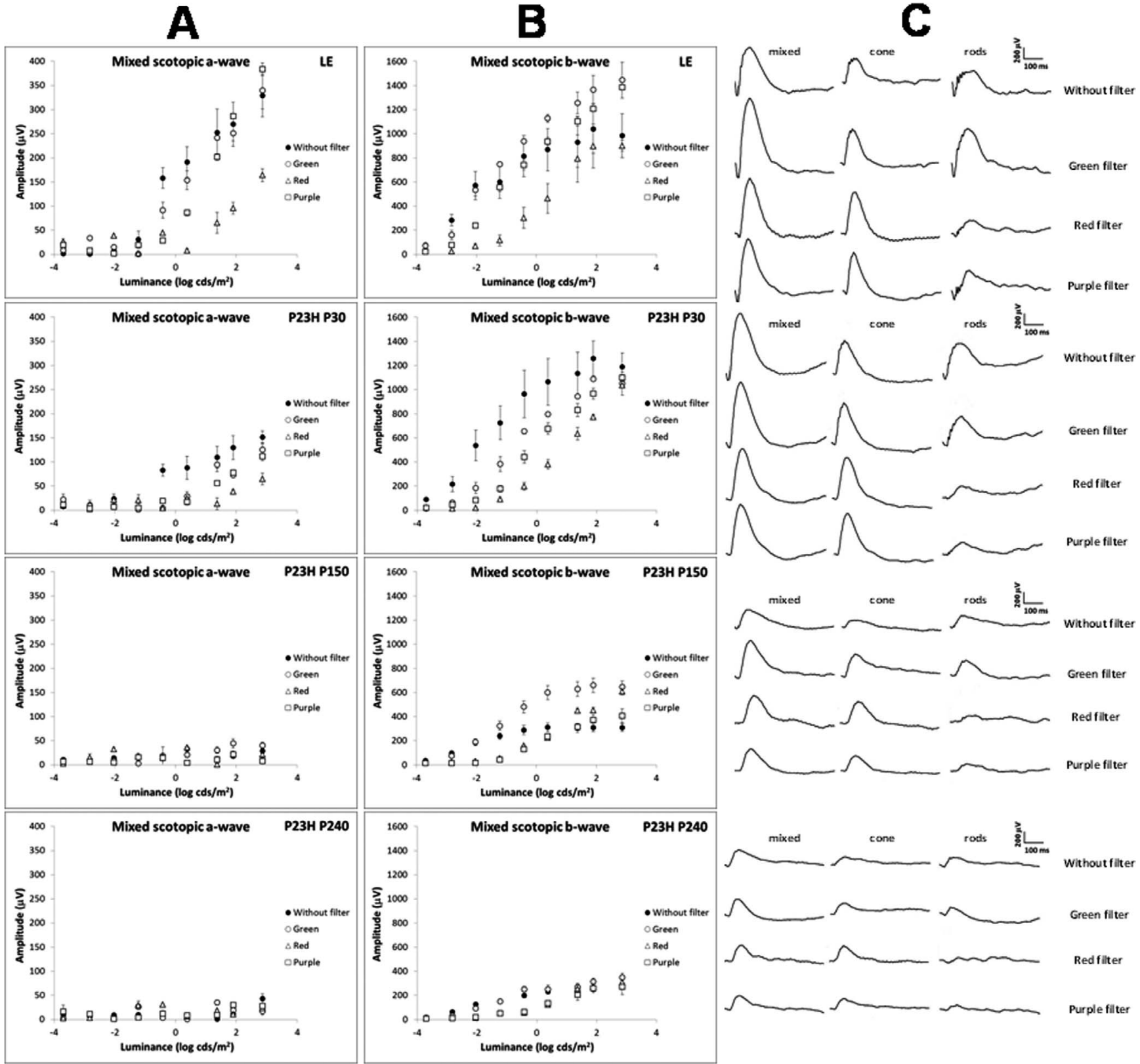

Figure 6. Electroretinographic amplitudes as a function of luminance and the application of the double flash protocol from LE (P90) and P23H rats (P30, P150, and P240) measured with different colored filters. For each group of rodents: ERG amplitude versus luminance series of mixed scotopic a-waves (A) and of mixed scotopic b-waves (B) from rats without filters (black circle), and with green (white circle), red (triangle), and purple (square) filters. Each point represents the mean of 4 rats. Vertical bars: SEM. (C) Application of the double flash protocol in rats without filters, and with green, red and purple filters. Left traces: mixed response obtained with a conditioning flash alone. Middle traces: isolated cone response obtained with a probe flash preceded by the conditioning flash. Right traces: rod response obtained by subtracting the isolated cone response from the mixed b-wave. Stimulus presentation is at beginning of each trace. Each trace represents the mean of 4 rats.

retina when rod damage is accelerated by raising ambient illumination. There is a close dependence of cone integrity on rod integrity, compared to the life-long persistence of cone function in the scotopic rat. ${ }^{32}$ Reducing environmental lighting $^{35}$ in combination with other treatments ${ }^{36}$ may delay retinal degeneration arising from rhodopsin mutations. Using green filters to repeatedly test the effect of therapeutic interventions in rats in vivo could minimize the light-induced damage. Greater age-related variations were obtained with green filters, as well as larger values, so significant changes could be easily distinguished from statistical deviations (Fig. 7).
The decreased ERG response with age also was clear, as already has been described (Fig. 5). The a-wave change was particularly clear, and the a-wave appears to be a better parameter than the b-wave to judge the efficacy of therapeutic manipulation in this rat model of retinal disease generated by photoreceptors. ${ }^{10,18}$ Values of $304 \pm 34 \mu \mathrm{V}$ for the LE rats and $113 \pm 10 \mu \mathrm{V}$ (P30), $25 \pm 5 \mu \mathrm{V}$ (P150), and $<20 \mu \mathrm{V}$ (no response, $\mathrm{P} 240$ ) for the $\mathrm{P} 23 \mathrm{H}$ rats were consistent with the gradual loss of photoreceptors. So, the long-term survival of some cones in transgenic rats could not be responsible for the minimum values of VA and CS, considering the ERG results. Thus, minimum OKT thresholds are achievable in pigmented 


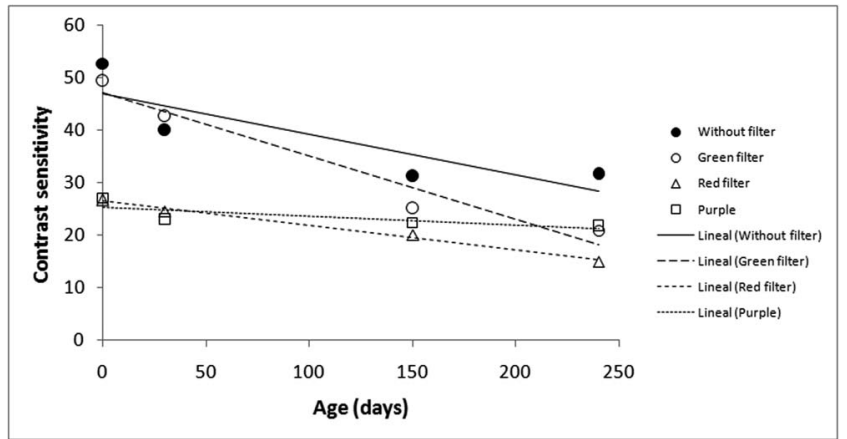

Figure 7. Contrast sensitivity (at a frequency of 0.089 cycles/deg) as a function of age in $\mathrm{P} 23 \mathrm{H}$ rats measured without filters (black circle) and with colored filters: green (white circle), red (triangle), and purple (square). Each point represents the mean of 8 rats. Lines of fit are plotted: without filters (continuous line), and green (medium dashed line), red (dashed line), and purple filters (dotted line).

$\mathrm{P} 23 \mathrm{H}$ rats without a full complement of photoreceptors, and it appears that rods and cones may not be necessary for minimum values of VA and CS, as others have concluded. ${ }^{37}$

In $\mathrm{P} 23 \mathrm{H}$ rats, there were no marked differences in most of the a- and b-waves with different colored filters (Figs. 6A, 6B), except in the P30 a-wave and the P150 b-wave, which could be related to the smaller measurements with the red filter and without a filter, respectively. In LE rats, a similar pattern between $\mathrm{a}$ - and $\mathrm{b}$-waves and colored filters was not found (Figs. 6A, 6B). However, the worst results were given by red filter, which has been shown to be the most limiting in the photoreceptor response.

The results of the ERG recordings obtained by the application of the double flash protocol showed differences in all groups (Fig. 6C). A-waves of rod-driven responses from $\mathrm{P} 23 \mathrm{H}$ rats were negligible $(<20 \mathrm{mV})$ using red and purple filters for all ages. In LE rats, smaller results were found using the same filters. Moreover, there were differences in the roddriven contribution to mixed scotopic b-waves, with larger values with green filters $(>40 \%)$ and without filters $(>50 \%)$ when compared to red and purple filters $(<33 \%)$. These results, although not conclusive, are in agreement with the filter characteristics. Using more animals to study each filter, evaluating the possible influence of the anesthesia, ${ }^{28}$ and choosing filters with a more suitable transmittance curve could allow a more detailed analysis.

In conclusion, visual function parameters decrease with age in pigmented $\mathrm{P} 23 \mathrm{H}$ rats. Irrespective of luminance, color filter, and retinal degeneration, minimum thresholds of visual acuity and contrast sensitivity were found. Smaller differences than expected were found using color filters, which might reflect that color differences are of minor contribution for rat vision. Responses to functional testing at long wavelengths were observed, where there is very low photoreceptor spectral sensitivity. The use of filters with functional testing could minimize light-induced retinal damage in rats.

\section{Acknowledgments}

Supported by the Spanish Ministry of Health ISCIII (PI13/01124, PS0901854), DGA Group B99, ISCIII RETICS RD12/0034/0010, and by a Zaragoza University Grant FPUZ-2011-BIO-02 (FS).

Disclosure: F. Segura, None; A. Sánchez-Cano, None; S. Jarabo, None; C. López de la Fuente, None; N. Cuenca, None; M.P. Villegas-Pérez, None; I. Pinilla, None

\section{References}

1. Walls GL. The visual cells of the white rat. J Comp Psychol. 1934;18:363-366.

2. Szél Á, Röhlich P, Caffé AR, Van Veen T. Distribution of cone photoreceptors in the mammalian retina. Microsc Res Tech. 1996;35:445-462.

3. Jacobs GH, Fenwick JA, Williams GA. Cone-based vision of rats for ultraviolet and visible lights. J Exp Biol. 2001;204:2439-2446.

4. Szél Á, Röhlich P. Two cone types of rat retina detected by antivisual pigment antibodies. Exp Eye Res. 1992;55:47-52.

5. Jacobs GH, Williams GA, Fenwick JA. Influence of cone pigment coexpression on spectral sensitivity and color vision in the mouse. Vision Res. 2004;44:1615-1622.

6. Hartong DT, Berson EL, Dryja TP. Retinitis pigmentosa. Lancet. 2006;368:1795-1809.

7. Congdon N. Causes and prevalence of visual impairment among adults in the United States. Arch Ophthalmol. 2004; 122:477-485.

8. Dryja TP. Retinitis pigmentosa and stationary night blindness. In: Scriver C, Beaudet A, Sly W, Valle D. The Metabolic and Molecular Bases of Inherited Disease. New York: McGrawHill; 2001:5903-5933.

9. Wilson JH, Wensel TG. The nature of dominant mutations of rhodopsin and implications for gene therapy. Mol Neurobiol. 2003;28:149-158.

10. Cuenca N, Fernández-Sánchez L, Sauvé Y, et al. Correlation between SD-OCT, immunocytochemistry and functional findings in an animal model of retinal degeneration. Front Neuroanat. 2014;8:151.

11. Roberts JE. Ocular photoxicity. J Photochem Photobiol B. 2001;64:136-143

12. Organisciak DT, Vaughan DK. Retinal light damage: mechanisms and protection. Prog Retin Eye Res. 2010;29:113-134.

13. LaVail MM, Gorrin GM, Repaci MA, Thomas LA, Ginsberg HM. Genetic regulation of light damage to photoreceptors. Invest Ophthalmol Vis Sci. 1987;28:1043-1048.

14. LaVail MM, Gorrin GM, Repaci MA. Strain differences in sensitivity to light-induced photoreceptor degeneration in albino mice. Curr Eye Res. 1987;6:825-834.

15. Naash MI, LaVail MM, Anderson RE. Factors affecting the susceptibility of the retina to light damage. Prog Clin Biol Res. 1989;314:513-522.

16. LaVail MM, Gorrin GM, Repaci MA, Yasumura D. Light-induced retinal degeneration in albino mice and rats: Strain and species differences. Prog Clin Biol Res. 1987;247:439-454.

17. Vaughan DK, Coulibaly SF, Darrow RM, Organisciak DT. A morphometric study of light-induced damage in transgenic rat models of retinitis pigmentosa. Invest Ophthalmol Vis Sci. 2003;44:848-855.

18. Machida S, Kondo M, Jamison JA, et al. P23H rhodopsin transgenic rat: correlation of retinal function with histopathology. Invest Ophthalmol Vis Sci. 2000;41:3200-3209.

19. Pinilla I, Lund RD, Sauvé Y. Enhanced cone dysfunction in rats homozygous for the $\mathrm{P} 23 \mathrm{H}$ rhodopsin mutation. Neurosci Lett. 2005;382:16-21.

20. Cuenca N, Pinilla I, Sauvé Y, Lu B, Wang S, Lund RD. Regressive and reactive changes in the connectivity patterns of rod and cone pathways of $\mathrm{P} 23 \mathrm{H}$ transgenic rat retina. Neuroscience. 2004; 127:301-317.

21. Prusky GT, Alam NM, Beekman S, Douglas RM. Rapid quantification of adult and developing mouse spatial vision using a virtual optomotor system. Invest Ophthalmol Vis Sci. 2004;45:4611-4616.

22. Douglas RM, Alam NM, Silver BD, Mcgill TJ, Tschetter WW, Prusky GT. Independent visual threshold measurements in the 
two eyes of freely moving rats and mice using a virtual-reality optokinetic system. Vis Neurosci. 2005;22:677-684.

23. Prusky GT, Alam NM, Douglas RM. Enhancement of vision by monocular deprivation in adult mice. $J$ Neurosci. 2006;26: 11554-11561.

24. Umino Y, Everhart D, Solessio E, et al. Hypoglycemia leads to age-related loss of vision. Proc Natl Acad Sci U S A. 2006;103: 19541-19545.

25. Alexander JJ, Umino Y, Everhart D, et al. Restoration of cone vision in a mouse model of achromatopsia. Nat Med. 2007;13: 685-687.

26. McGill TJ, Lund RD, Douglas RM, et al. Syngeneic Schwann cell transplantation preserves vision in RCS rat without immunosuppression. Invest Ophthalmol Vis Sci. 2007;48:1906-1912.

27. Radlwimmer FB, Yokoyama S. Genetic analyses of the green visual pigments of rabbit (oryctolagus cuniculus) and rat (rattus norvegicus). Gene. 1998;218:103-109.

28. Nair G, Kim M, Nagaoka T, et al. Effects of common anesthetics on eye movement and electroretinogram. Doc Ophthalmol. 2011;122:163-176.

29. Pinilla I, Lund RD, Sauvé Y. Contribution of rod and cone pathways to the dark-adapted electroretinogram (ERG) b-wave following retinal degeneration in RCS rats. Vision Res. 2004; 44:2467-2474.

30. Jacobs GH, Neitz J, Deegan JF II Retinal receptors in rodents maximally sensitive to ultraviolet light. Nature. 1991;353:655656.

31. Chrysostomou V, Stone J, Valter K. Life history of cones in the rhodopsin-mutant p23h-3 rat: Evidence of long-term survival. Invest Ophthalmol Vis Sci. 2009;50:2407-2416.

32. Chrysostomou V, Valter K, Stone J. Cone-rod dependence in the rat retina: variation with the rate of rod damage. Invest Ophthalmol Vis Sci. 2009;50:3017-3023.

33. Umino Y, Solessio E, Barlow RB. Speed, spatial, and temporal tuning of rod and cone vision in mouse. J Neurosci. 2008;28: 189-198.

34. Chrysostomou V, Stone J, Stowe S, Barnett NL, Valter K. The status of cones in the rhodopsin mutant $\mathrm{P} 23 \mathrm{H}-3$ retina: lightregulated damage and repair in parallel with rods. Invest Ophthalmol Vis Sci. 2008;49:1116-1125.

35. Organisciak DT, Darrow RM, Barsalou L, Kutty RK, Wiggert B. Susceptibility to retinal light damage in transgenic rats with rhodopsin mutations. Invest Ophthalmol Vis Sci. 2003;44: 486-492.

36. Fernández-Sánchez L, Lax P, Pinilla I, Martín-Nieto J, Cuenca N. Tauroursodeoxycholic acid prevents retinal degeneration in transgenic P23H rats. Invest Ophthalmol Vis Sci. 2011;52: 4998-5008.

37. McGill TJ, Prusky GT, Douglas RM, et al. Discordant anatomical, electrophysiological, and visual behavioral profiles of retinal degeneration in rat models of retinal degenerative disease. Invest Ophthalmol Vis Sci. 2012;53:6232-6244. 